\title{
Two-Dimensional Nanomaterials for Peripheral Nerve Engineering: Recent Advances and Potential Mechanisms
}

\author{
Zhiwen Yan ${ }^{1,2,3+}$, Cheng Chen ${ }^{1 \dagger}$, Gonzalo Rosso ${ }^{4,5,6 *}$, Yun Qian ${ }^{1,2,3 *}$ and Cunyi Fan ${ }^{1,2,3 *}$ \\ ${ }^{1}$ Department of Orthopedics, Shanghai Jiao Tong University Affiliated Sixth People's Hospital, Shanghai, China, ${ }^{2}$ Shanghai \\ Engineering Research Center for Orthopaedic Material Innovation and Tissue Regeneration, Shanghai, China, ${ }^{3}$ Youth Science and \\ Technology Innovation Studio, Shanghai Jiao Tong University School of Medicine, Shanghai, China, ${ }^{4}$ Max Planck Institute for the \\ Science of Light, Erlangen, Germany, ${ }^{5}$ Max-Planck-Zentrum für Physik und Medizin, Erlangen, Germany, ${ }^{6}$ Institute of Physiology \\ II, University of Münster, Münster, Germany
}

OPEN ACCESS

Edited by:

Jianxun Ding,

Changchun Institute of Applied Chemistry (CAS), China

Reviewed by:

Francesco Baino,

Politecnico di Torino, Italy

Wen Shi,

University of Nebraska Medical

Center, United States

*Correspondence:

Gonzalo Rosso

gonzalo.rosso@mpl.mpg.de

Yun Qian

Iollipopcloudland@foxmail.com

Cunyi Fan

cyfan@sjtu.edu.cn

${ }^{+}$These authors have contributed equally to this work

Specialty section: This article was submitted to Biomaterials,

a section of the journal Frontiers in Bioengineering and

Biotechnology

Received: 23 July 2021 Accepted: 19 October 2021 Published: 08 November 2021

Citation:

Yan Z, Chen C, Rosso G, Qian Y and

Fan C (2021) Two-Dimensional Nanomaterials for Peripheral Nerve Engineering: Recent Advances and

Potential Mechanisms.

Front. Bioeng. Biotechnol. 9:746074.

doi: 10.3389/fbioe.2021.746074
Peripheral nerve tissues possess the ability to regenerate within artificial nerve scaffolds, however, despite the advance of biomaterials that support nerve regeneration, the functional nerve recovery remains unsatisfactory. Importantly, the incorporation of twodimensional nanomaterials has shown to significantly improve the therapeutic effect of conventional nerve scaffolds. In this review, we examine whether two-dimensional nanomaterials facilitate angiogenesis and thereby promote peripheral nerve regeneration. First, we summarize the major events occurring after peripheral nerve injury. Second, we discuss that the application of two-dimensional nanomaterials for peripheral nerve regeneration strategies by facilitating the formation of new vessels. Then, we analyze the mechanism that the newly-formed capillaries directionally and metabolically support neuronal regeneration. Finally, we prospect that the two-dimensional nanomaterials should be a potential solution to long range peripheral nerve defect. To further enhance the therapeutic effects of two-dimensional nanomaterial, strategies which help remedy the energy deficiency after peripheral nerve injury could be a viable solution.

Keywords: two-dimensional nanomaterial, peripheral nerve regeneration, nerve scaffold, nerve tissue engineering, angiogenesis, nanomedicine

\section{TWO-DIMENSIONAL NANOMATERIALS AND PERIPHERAL NERVE ENGINEERING}

Two-dimensional (2D) nanomaterials have received great interest by the whole research community due to their exceptional electrochemical properties, based on the special character of atom-scale thickness, which allows for the free transfer of electrons on the material surface. Furthermore, 2D nanomaterials exhibit enhanced and tunable electronic, physical and chemical properties due to their distinctive phase, crystallinity, degree of exfoliation, stability and fundamental limitation of thickness (Rohaizad et al., 2021). In addition, as it is the case for the well-known electroactive nature of peripheral nerve tissue (Yao et al., 2021), 2D nanomaterials possess remarkable electrical properties making them ideal candidates for improving the outcomes of peripheral nerve injuries (PNI) (Qian et al., 2019d). Although there are several exciting studies demonstrating that the $2 \mathrm{D}$ nanomaterial-based neural regeneration devices could improve the efficiency of peripheral nerve regeneration (PNR) (Table 1) the exact mechanisms underlying this phenomenon remains elusive. 
TABLE 1 | Therapeutic effects of the 2D nanomaterial functionalized nerve scaffold in vivo.

\begin{tabular}{|c|c|c|c|c|c|c|c|}
\hline 2D Nano-material & $\begin{array}{c}\text { Concentration } \\
(\%)\end{array}$ & $\begin{array}{c}\text { Nerve } \\
\text { defect } \\
\text { range }(\mathrm{mm})\end{array}$ & $\begin{array}{c}\text { Time after } \\
\text { implantation } \\
\text { (weeks) }\end{array}$ & $\begin{array}{c}\mathrm{NCV} \\
\left(\mathrm{ms}^{-1}\right)\end{array}$ & $\begin{array}{l}\text { DCMAP } \\
(\mathrm{mV})\end{array}$ & $\begin{array}{l}\text { Angiogenesis } \\
\text { marker }\end{array}$ & Ref. \\
\hline Graphene oxide & 1 & 15 & 18 & 33.4 & 25.1 & CD31, CD34 & $\begin{array}{l}\text { Qian et al. } \\
(2018 b)\end{array}$ \\
\hline Graphene oxide & / & 10 & 8 & 24.8 & 9.9 & l & $\begin{array}{l}\text { Zhang et al. } \\
(2020)\end{array}$ \\
\hline $\begin{array}{l}\text { Carboxylic graphene } \\
\text { oxide }\end{array}$ & l & 10 & 12 & 39 & 3.8 & l & $\begin{array}{l}\text { Chen et al. } \\
(2019)\end{array}$ \\
\hline $\begin{array}{l}\text { Reduced graphene } \\
\text { oxide }\end{array}$ & 1.14 & 10 & 12 & 25.0 & / & l & $\begin{array}{l}\text { Wang et al. } \\
(2019)\end{array}$ \\
\hline $\begin{array}{l}\text { Reduced graphene } \\
\text { oxide }\end{array}$ & 0.5 & 10 & 12 & 25 & 2.5 & / & Fang et al. (2020) \\
\hline Graphene & 4 & 18 & 18 (months) & 42.9 & 34.5 & CD34, VEGF & $\begin{array}{l}\text { Qian et al. } \\
\text { (2021a) }\end{array}$ \\
\hline Graphene & 5 & 10 & 6 & / & 5.6 & Histology & Lee et al. (2020) \\
\hline Black phosphorus & 0.5 & 20 & 16 & 29.5 & 22.1 & VEGF, CD34 & $\begin{array}{l}\text { Qian et al. } \\
(2019 b)\end{array}$ \\
\hline Zinc oxide & 10 & 15 & 18 & 45.4 & 22.6 & VEGF, CD34 & Qian et al. (2020) \\
\hline Boron nitride & 10 & 15 & 18 & 48.2 & 30.2 & CD34 & $\begin{array}{l}\text { Qian et al. } \\
(2021 b)\end{array}$ \\
\hline
\end{tabular}

NVC, Nerve conduction velocity; DCMAP, Distal compound motor action potential; VEGF, Vascular endothelial growth factor; CD, Cluster differentiation; I: Not applicable.

In this review, we summarize the applications of $2 \mathrm{D}$ nanomaterials in aiding PNR and focus on the mechanism of new-vessel guided regeneration. First, we summarize the major physiological events occurring after PNI. Second, we describe the application of $2 \mathrm{D}$ nanomaterials in peripheral nerve engineering and the corresponding therapeutic effects. Third, we analyze the mechanism by that the newly-formed capillaries provide directionality and metabolic support for neural regeneration. Finally, we discuss the use of $2 \mathrm{D}$ nanomaterial-based neural regeneration devices as a potential biomedical strategy to improve long range peripheral nerve defects, and how that could help to remedy the energy deficiency after PNI.

\section{PERIPHERAL NERVE DAMAGE AND REGENERATION}

The peripheral nerve acts as a real-time information transmitter between human brain and the rest body. Compared to the wellprotected brain and spinal cord in the central nervous system by the skull and vertebrates, peripheral nerves are not surrounded by hard structures, making them extremely vulnerable to different physical damage. Normally, PNIs result in numbness, locomotor dysfunction and even life-long disabilities for individuals (Figure 1A), causing huge social and economic burdens (Taylor et al., 2008). The gold standard treatment to PNI (anastomosis and autograft transplanting) have intrinsic limitations such as donor site morbidity and size mismatch (Ray and Mackinnon, 2010). Despite researchers have developed various neural regeneration devices as alternative therapies to PNIs, these products fail to guarantee satisfactory functional nerve recovery.
The peripheral nervous system (PNS) is among the very few tissues in adult mammals which possess remarkable regeneration capabilities after injury. After PNI, Schwann cells-the myelin forming cells in the PNS-dedifferentiate and transform into a repair phenotype that play key roles in the nerve regeneration process (Jessen and Mirsky, 2016; Clements et al., 2017). However, such regeneration process is often mistakenly thought to occur robustly and successfully (Scheib and Höke, 2013). Hence, the complex biological events induced by the injury lead to changes in the biochemical and mechanical properties of the nerve tissue microenvironment, impairing the restoration of nerve function (Yan and Qian, 2020; Qian et al., 2021c). Therefore, it is of tremendous biomedical interest to improve current strategies to regenerate peripheral nerves more efficiently, especially in nerve defect models.

PNIs are characterized by the retraction of two nerve stumps or the direct loss of a nerve segment. During the regeneration process, a natural bridge forms between the two nerve stumps (Min et al., 2021) where damaged axons need to grow through in order to reach the distal nerve trunk and their designated target effectors. The distal trunk, usually referred to as Bands of Büngner, provides a pro-regenerative environment for the outgrowth of axons across the lesion site. However, the environment within the nerve bridge is rather hostile for Schwann cell and axons to travel through. Endothelial cells, which help form new vessels, help remodel the hostile environment and play an essential and inevitable role in the regeneration process (Cattin and Lloyd, 2016). The degree of vascularization determines the success of PNR, i.e., the higher degree of vascularization within the nerve bridge contributes to better PNI outcomes while the inhibition of vascularization destroys normal PNR. 


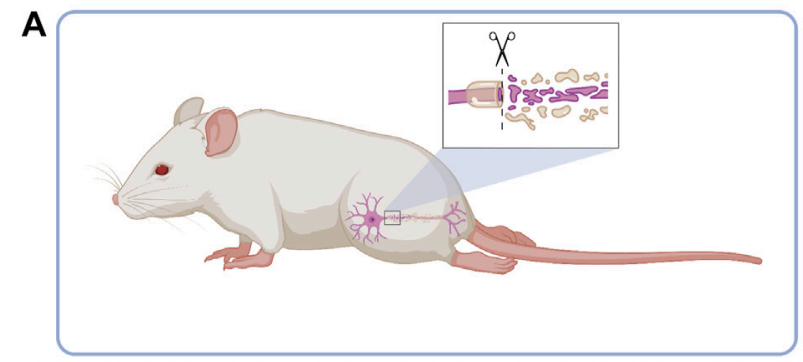

B

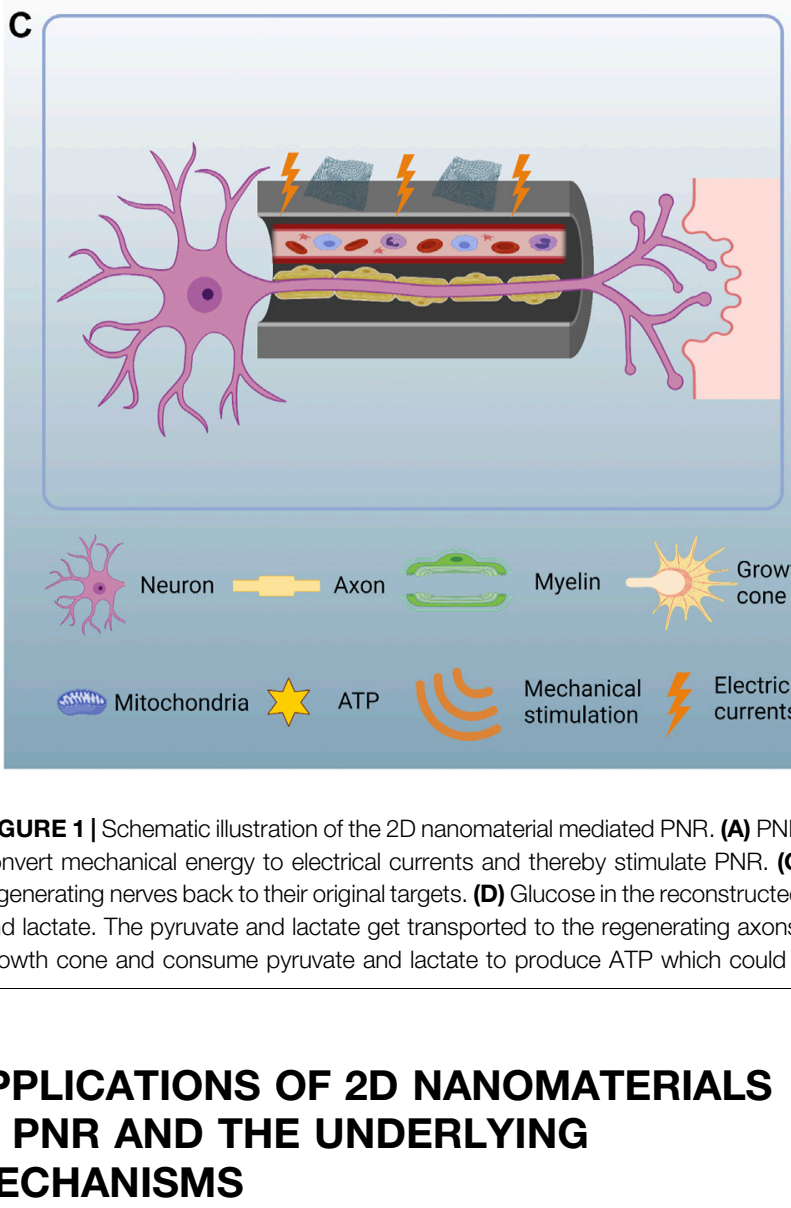

In this subsection, we update the applications of $2 \mathrm{D}$ nanomaterials for the fabrication of nerve scaffolds, and focus on the mechanism that the $2 \mathrm{D}$ nanomaterials could facilitate the formation of new blood-vessels and discuss their pro-regenerative effects for PNR (Figure 1B).

\section{Graphene}

Graphene is the most representative material of the $2 \mathrm{D}$ nanomaterial family. The unique nano-scale honeycomb planar structure formed by carbon $\mathrm{sp}^{2}$ hybridization, provides graphene with outstanding electrical conductivity, surface area and mechanical properties (Qian et al., 2018c). Convertino et al. observed that dorsal root ganglion (DRG) neurons elongate more when cultured on graphene surfaces compared to glass in vitro (Convertino et al., 2020). The authors attribute this effect to the decreased retrograde transport of nerve growth factor (NGF), and

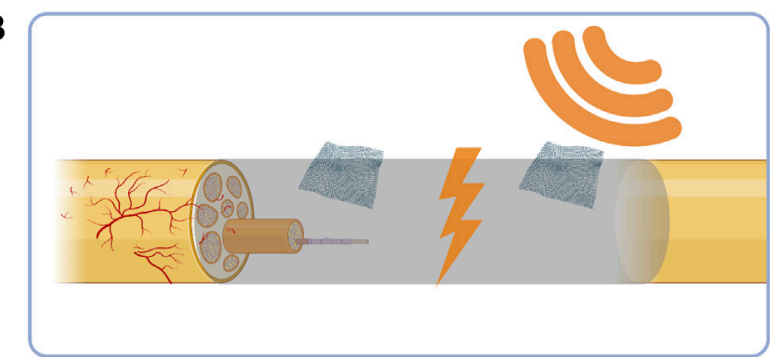

D

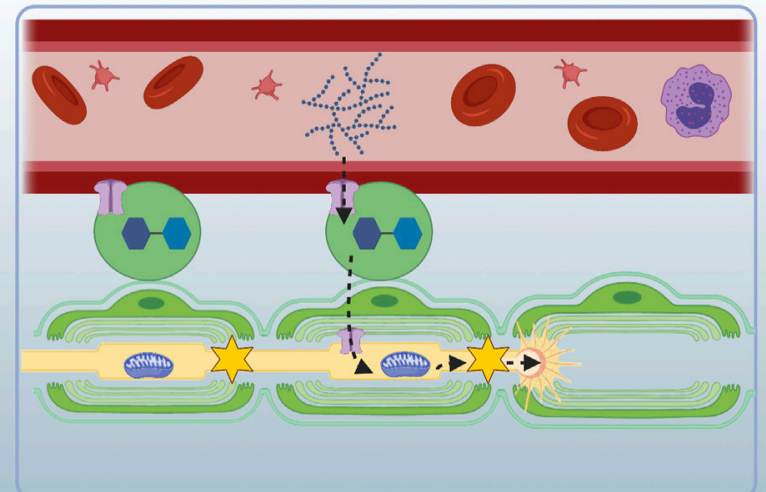


axons resides in spinal cords and DRGs, so we dissected the spinal cords and DRGs of the rats implanted with graphene-based scaffolds 18 months after injury. We found that the expression level of nestin, a protein specifically expressed in neural stem cells that plays essential roles in neural stem cell differentiation, were upregulated. These results indicate that the presence of graphene accelerates axonal regrowth by creating a pro-angiogenesis microenvironment and increase the stemness of neurons. Interestingly, the high VEGF concentration within the graphene scaffold chamber is also responsible for the increased neuronal activity after PNI. The receptors of VEGF are not only expressed on endothelial cells but also present on the axons and growth cones. The conditional knockdown of hypoxia-inducible factor 1a (HIF-1a) in mice DRG leads to impaired neuronal regenerative ability and the local administration of VEGF could partly remedy this (Cho et al., 2015). Sondell et al. also pointed out VEGF stimulates PNR by acting both on the growing axons and cell bodies (Sondell et al., 2000). Therefore, the graphene induced VEGF expression is responsible for both local angiogenesis and neuronal regeneration.

\section{Graphene Oxide}

The incorporation of graphene oxide, an extremely oxidized graphene derivative, elevated the electrical conductivity of a polymeric conduit to $4.55 \times 10^{4} \mathrm{~S} \mathrm{~cm}^{-1}$ while the nonoxidized single-layered graphene conduit is $8.92 \times 10^{-3} \mathrm{~S}$ $\mathrm{cm}^{-1}$. We have tested the neural functional recovery from the aspect of electrophysical regain, remyelination degree and morphological regeneration at $6,12,18$ weeks after implantation (Qian et al., 2018a). Interestingly, the graphene oxide embedded nerve guide shows superior regenerative potential compared to the polymeric counterparts at various time points. Of note, after 18 weeks, the regeneration outcome of graphene oxide containing scaffold was similar to that of the clinical "gold standard" autograft transplantation. Then we proposed that this phenomenon could be due to the proangiogenesis effects of graphene oxide. As expected, the microvessel density and vessel-like structure area significantly elevated in the graphene oxide containing conduit. Further, the CD34, a transmembrane phosphor-glycoprotein, widely used as biomarker of hematopoietic progenitor cells, significantly increased by the controlled release of graphene oxide (Sidney et al., 2014). Additional results showed that AKT-eNOS-VEGF signaling pathway involved in the increase in endothelial cell proliferation may provide new insights for the underlying physiological mechanisms of graphene function in nerve regeneration.

\section{Black Phosphorus}

Another promising 2D nanomaterials that has recently received tremendous attention is the black phosphorus for its thicknessdependent bandgap, high charge-carrier mobility, in-plane anisotropic structure, and biodegradable properties (Tao et al., 2015). As a new star of the 2D materials family, black phosphorus exhibited huge potential in aiding peripheral nerve regrowth. For example, the incorporation of $0.5 \%$ black phosphorus into polymers elevates the electrical conductivity to $9.81 \times 10^{-3} \mathrm{~S}$ $\mathrm{cm}^{-1}$. Hence, a black phosphorus containing nerve scaffold restores the bioelectrical continuity of damaged nerves and promotes the expression of VEGF, thereby contributing to successful formation of new vessels (Qian et al., 2019b). VEGF, as described above, specifically targets endothelial cells and is essential for vasculogenesis. In a rat long-range nerve defect model $(20 \mathrm{~mm})$, the incorporation of black phosphorus into a nerve guide has shown to successfully enhance the formation of vessels compared to the polymeric counterparts. The elevated vessel density is responsible for faster neural regeneration. Therefore, the function of target muscle measured by the distal compound motor action potential (DCMAP) increased from 10.1 to $22.1 \mathrm{mV}$ in the polymer group compared to the black phosphorus group 4 months after implantation.

Moreover, mounting evidence suggest that $2 \mathrm{D}$ nanomaterials could facilitate PNR by generating electrical currents. Conversely, the process of angiogenesis has also been linked to these $2 \mathrm{D}$ materials. Electrical currents that enhance axonal growth combined with the formation of new vessels are two promising outcomes with tremendous potential for the advancement of nerve regeneration strategies. Electrical stimulation has long been proved to be a valid therapeutic strategy for accelerating nerve regrowth (Qian et al., 2019a) and researchers have developed artificial electrical stimulators to facilitate nerve regeneration (Koo et al., 2018). However, such equipment is usually cumbersome to use because it requires external power sources and the implantation of electrodes is also prone to trigger neuroinflammation and gliosis (Cheng et al., 2020). Back in 1991, Fine et al. proposed the application of vinylidenefluoride-trifluoroethylene, a conventional piezoelectrical active material utilized in the engineering of neural regeneration devices (Fine et al., 1991). The piezoelectrical active material possesses the ability to transform the mechanic energy into electrical stimulation. Basically, when the piezoelectrical active neural scaffold gets mechanically deformed by an internal stimulation (e.g., muscle compression) or an external stimulation (e.g., ultrasound), the electrical currents are generated on the scaffold's surface, thereby providing a wireless and self-powered nerve electrical stimulation therapy. Unfortunately, this innovative experiment encountered some difficulties. The polymeric piezoelectric material was hard to degrade in vivo and some fibrous capsules formed around the scaffold (Reis et al., 2012). From a crystallography point of view, except for the cubic class 432, all non-centrosymmetric point groups possess the piezoelectric effect. With the fast development of $2 \mathrm{D}$ material synthesis technology, the reduced $2 \mathrm{D}$ dimensionality embodies spontaneous breakdown of threedimensional (3D) symmetry. Therefore, some nonpiezoelectric bulk materials may become piezoelectric when thinned to single atomic layer (Lin et al., 2018). In addition, the $2 \mathrm{D}$ piezoelectric nanomaterials have the advantage of possessing ultra-high piezoelectric coefficients and the ability to degraded by human enzymes (Fei et al., 2015; Lin et al., 2017).

\section{Zinc Oxide}

Interestingly, it has been shown that the incorporation of $2 \mathrm{D}$ zinc oxide embedded in a polymeric conduit is able to bridge a sciatic 
nerve defect (Qian et al., 2020a). Qian and colleagues took advantage of a treadmill to trigger electrical activity of the zinc oxide nerve implants in rats. Results from these experiments showed that after $30 \mathrm{~min}$ running exercise per day during 18 weeks, the zinc oxide group showed significantly improved nerve regeneration outcomes compared to controls animals. Hence, the zinc oxide nerve scaffold promoted the expression of $\mathrm{S} 100$ and myelin basic protein (MBP) in regenerated nerve tissues, indicating a higher number of Schwann cells and myelinated axons. In addition, the $\beta 3$-tubulin and NF160 levels were also elevated, indicating an increased density of regrowing axons. The cellular and molecular mechanisms underlying nerve regeneration in zinc oxide nerve implants remains still poorly understood. However, an in vitro study showed that the ultrasonic activated piezoelectric scaffold increased the VEGF secretion in Schwann cells. On the other hand, an in vivo study demonstrated that the exercise activated piezoelectric therapy up-regulates the VEGF and CD34 expression levels within the scaffold chamber (Qian et al., 2020).

\section{Boron Nitride}

The incorporation of $2 \mathrm{D}$ boron nitride into nerve scaffolds has also shown promising results for the treatment of PNIs. Structured boron nitride possesses unique properties, such as an atomically flat surface, free of dangling bonds, charged impurities, highly chemical stability, superior elastic modulus and outstanding mechanical flexibility (Zhang et al., 2021). Owing to the polarization of the $\mathrm{B}-\mathrm{N}$ chemical bond, the $2 \mathrm{D}$ boron nitride exhibits piezoelectrical activity. Qian et al. incorporated boron nitride into a nerve scaffold to test the neuronal regeneration ability of this $2 \mathrm{D}$ nanomaterial (Qian et al., 2021b). The authors first validated that the 2D boron nitride scaffold possessed excellent piezoelectric property measured by piezo-response force microscopy. Then, they applied a treadmill running protocol to induce mechanical deformation on the 2D boron nitride scaffold, thereby generated electrical currents. 18 weeks post implantation, the morphology of regenerated nerve tissues was analyzed by transmission electron microscopy and found a fully regenerated peripheral nerve comprised of axons and myelin sheaths. Specifically, the axons inside the $2 \mathrm{D}$ boron nitride scaffold showed an increase of axonal areas, myelin sheath thickness, diameter of myelinated axons, and number of myelinated axons. Furthermore, the locomotor function restoration measured by walking track analysis and DCMAP was improved. The motor function restoration is a challenge for artificial nerve scaffold due to the denervation induced muscle atrophy. The $2 \mathrm{D}$ boron nitride scaffold preserved the endplate function and promoted the muscle fiber phenotype shift from slow muscle fiber to fast muscle fiber. The faster axonal regeneration was responsible for this phenomenon and as the micro vessel-reconstruction was the premise for axonal regeneration. As expected, CD34, the indicator of neo-vessel formation, was significantly increased in the $2 \mathrm{D}$ boron nitride scaffold group.

\section{D NANOMATERIALS FACILITATE MICRO-VESSEL FORMATION AND PROVIDE GUIDANCE FOR MIGRATING SCHWANN CELLS}

It remains controversial whether $2 \mathrm{D}$ nanomaterials could enhance the angiogenesis ability of endothelial cells. Cibecchini et al. reported that the 2D graphene oxide compromised the angiogenic potential of primary human endothelial cells in vitro when administered at high concentration $\left(50 \mu \mathrm{g} \mathrm{ml}^{-1}\right)$ (Cibecchini et al., 2020). The excessive amount of graphene oxide internalized into the cells forms aggregates and affects the consumption of niacinamide. Excessive graphene oxide hindered angiogenesis of human endothelial cells by altering the distribution of mitochondria and disturbing several metabolic pathways. Contrary to in vitro results, in vivo data showed that $2 \mathrm{D}$ nanomaterials stimulate the formation new vessels in different disease models (Qian et al., 2019d; Norahan et al., 2019; Wierzbicki et al., 2020). One possible explanation for this phenomenon could be that in vivo studies utilized scaffolds which allow for the controlled release of $2 \mathrm{D}$ nanomaterials. But more importantly, in vivo environment possesses the multi-cellular complexity. Macrophages, as the important line of defense in human body, are extremely sensitive to foreign implants such as $2 \mathrm{D}$ nanomaterials. Xue et al. pointed out that $2 \mathrm{D}$ graphene oxide nanosheets could be phagocytosed by macrophages and activate the toll-like receptors (TLR)/myeloid differentiation factor 88 (MyD88)/nuclear factor kappa-B (NF- $\mathrm{kB}$ ) pathway, thereby excrete VEGF (Xue et al., 2018). The human umbilical vein endothelial cells (HUVEC) treated with graphene oxide conditioned macrophage culture supernatant exhibited enhanced tube formation ability. Overall, the incorporation of 2D nanomaterials could increase new vessel formation in vivo. In the following section we discuss the mechanism behind the micro-vessel mediated PNR.

Back in the 1990s, researchers started to observe the relevance between capillary number and nerve regeneration outcomes. Hobson et al. visualized the interactions between RECA-1 positive endothelial cells, S100 positive Schwann cells and axons at different time points after PNI (Hobson et al., 1997). They found first the sprouted of newly formed blood vessels, followed by the migration of Schwann cells and regenerating axons. Of note, the position of Schwann cells and axons within the nerve bridge never exceed the sprouting vessels. In a different work, the incorporation of the proliferative marker EdU in endothelial cells has been used to prove that the vessels are newly formed (Cattin et al., 2015) and observed that the EdU positive endothelial cells only existed within the regenerating nerves.

How does the endothelial cells respond to the nerve injury? In intact nerve tissues, blood vessels go along with nerve fascicles and supply oxygen and nutrients to maintain the normal physiology of nerve function. However, after traumatic nerve injury, both the nerves and the accompanied blood vessels are severely damaged and eventually completely transected causing a 
hypoxic microenvironment within the injured site. 2 days after nerve dissection, the injury site undergoes severe hypoxia (Cattin et al., 2015). Interestingly, among the diverse cell components of peripheral nerves, macrophages are extremely sensitive to hypoxia. Over 98\% hypooxyprobe-1 positive cells are macrophages and over $80 \%$ macrophages are hypoxic. Then, the hypoxic microenvironment stabilizes transcriptional factor HIF-1a and initiates the expression of downstream protein VEGF. The VEGF binds to its receptor on endothelial cells and stimulates endothelial cell proliferation which triggers the formation of new vessels and sprouts from the two ends of dissected nerves to form a vessel bridge.

What is the cellular mechanism behind the blood vessel guided nerve regeneration? Schwann cells, a key cell type that plays a major role in the orchestration of PNR, get attracted by the vessel bridge (Clements et al., 2017). The Schwann cells directly migrate along the vessel bridge in an amoeboid-like mode. The actomyosin cytoskeleton is responsible for this behavior, which is impaired by the application of Rho-kinase inhibitor. Besides, the aligned blood vessels provide directionality for Schwann cell migration and intentionally misdirection (implantation of VEGF releasing beads into the surrounding muscle beds) of endothelial cells results in completely failed PNR. Schwann cells follow the vessel track to form a permissive corridor for regenerating axons to travel through (Cattin et al., 2015). It has been shown that Netrin1/DCC signal acts as a critical cue for regenerating axons to grow alongside the migrating Schwann cells (Webber et al., 2011). Only after the regenerating axons reach their final targets, the peripheral nerve damage could be repaired and the denervation induced atrophy of target organs could be remedied.

Where are the newly formed vessels from? Cattin et al. suggested that the new vessels originate from both the proximal and distal nerve stumps, whereas Hobson et al. pointed out that new vessels originate from the adjacent muscle beds (Hobson et al., 1997; Cattin et al., 2015). The differences in the origin of vessels' formation could be explained by the different injury models utilized. Cattin et al. used the simple nerve dissection surgery while Hobson et al. implanted a $10 \mathrm{~mm}$ fibronectin graft.

In summary, newly formed vessel is the premise for successful PNR. The capillaries directly guide Schwann cell migration and indirectly contribute to axonal regeneration. The ability of angiogenesis modulation should be taken into account when designing a neural regeneration device (Figure 1C).

\section{THE 2D NANOMATERIAL FACILITATED MICRO-VESSEL FORMATION PROVIDES ENERGY FOR PROLIFERATING SCHWANN CELLS}

It remains an open question whether $2 \mathrm{D}$ nanomaterials facilitate micro-vessel formation and guidance to migrating Schwann cells. In this subsection, we propose a new mechanism which suggests that the newly-formed capillaries may provide nutrients necessary for the proliferation Schwann cells, and metabolically support neuronal regeneration.
Tissue regeneration is a highly energy-demanding process and energy deficiency happens during PNR as well (Han et al., 2016). In response to injury, the PNS adapts a series of metabolic changes to initiate the regeneration process. Recent studies revealed bioenergetically compensatory processes in neuronal axons and Schwann cells. Mitochondria, the cell power-house in mammalian cells, accumulate at the site of injury in axons by microtubulebased mitochondrial transport from the neuronal soma (Han et al., 2016; Patrón and Zinsmaier, 2016). On the other hand, after nerve injury Schwann cells undergo a glycolysis shift to synthesize excessive amount of pyruvate and lactate (Babetto et al., 2020; Trimarco and Taveggia, 2020). The pyruvate and lactate get transferred to the injured axons, serving as metabolic substrate to support the mitochondrial ATP synthesis (Patrón and Zinsmaier, 2016; Trimarco and Taveggia, 2020). In short, Schwann cells metabolically support axonal regeneration, however, where do Schwann cells get metabolic substrate is not completely understood A similar phenomenon occurs in the central nervous system (CNS) where astrocytes and oligodendrocytes - the glial cells in CNS - transfer lactate to neuronal axons and the lactate is used to generate metabolic energy in the form of ATP, thereby supporting the high energy consumption of neuronal axons (Lee et al., 2012; Rinholm and Bergersen, 2012). Of note, the astrocytes and oligodendrocytes break up blood glucose to generate lactate. In other words, blood vessels energetically support axons through glial cells. As mentioned in 2D Nanomaterials Facilitate Micro-Vessel Formation and Provide Guidance for Migrating Schwann Cells, the newly formed blood vessel is the premise for neural regeneration in PNS where the capillaries serve as tracks for Schwann cells to migrate along and indirectly guide axonal regeneration (Cattin et al., 2015). It remains open whether the newly formed vessels support axonal regeneration in other ways, for instance, by directly providing energy necessary for axonal regeneration. The latter $t$ is highly possible if we consider that the expression levels of glucose transporter 1 (GLUT1) is upregulated during the glycolysis shift in Schwann cells after PNI (Babetto et al., 2020). The GLUT1 is widely accepted as a protein that help glucose transfer between blood vessels and organs (Veys et al., 2020). Thus, the metabolic substrate Schwann cells use may possibly originated from the nearby blood vessels and angiogenesis supports neurogenesis not only by providing directionality but also by supplying nutrients (Figure 1D).

Therefore, apart from angiogenesis, attention should also be paid upon the metabolic process during PNR. However, several studies pointed out that the $2 \mathrm{D}$ nanomaterials pose threat on endothelial cells by disturbing the metabolic pathways. In this regard, Chen et al. performed transcriptional sequencing on $2 \mathrm{D}$ black phosphorus exposed aortic artery and identified metabolic disturbance (Chen et al., 2021). Furthermore, Luo et al. also reported that 2D graphene oxide impaired HUVEC viability by compromising lipid droplet biogenesis (Luo et al., 2021). Lipid, as an integral part of cellular membrane, is essential 
for endothelial cell proliferation. Importantly, the researchers found the addition of oleic acid and $\alpha$-linolenic acid, metabolic substrate in lipid biosynthesis, could alleviate the $2 \mathrm{D}$ graphene oxide induced cytotoxicity in HUVEC and restore the lipid biosynthesis. Overall, 2D nanomaterials facilitate angiogenesis, the newly formed vessels nourish sprouting axons via Schwann cells. However, the 2D nanomaterial itself potentially harms the cellular metabolism, while the incorporation of a biometabolic active substrate could remedy this and further enhance the therapeutic effects of $2 \mathrm{D}$ nanomaterials.

\section{FINAL REMARKS}

Artificial nerve scaffolds hold great promise for the biomedical treatment of injured nerves by connecting the damaged stumps and new nerve tissues form within the scaffold chamber (Liu et al., 2019; Jiang et al., 2020; Yan et al., 2020; Li et al., 2021). However, the functional restoration of PNIs still remains unsatisfactory (Qian et al., 2018b; Qian et al., 2019b; Qian et al., 2019c). The formation of new vessels is the premise for successful PNR, and augmentation of angiogenesis could significantly advance the quality of regenerated nerves and nerve repair strategies. 2D nanomaterials exhibit huge potential in aiding PNR. They not only promote axonal growth via electric stimulation, but also facilitate angiogenesis within the regenerating nerve tissue, which directly stimulates Schwann cell proliferation and enhance axonal outgrowth. In this review, we summarize the updates on the mechanism underlying this phenomenon and point out that the $2 \mathrm{D}$ nanomaterial promotes PNR via facilitating angiogenesis.

Despite there are strong evidences to support that $2 \mathrm{D}$ nanomaterials modulate the angiogenesis ability to aid PNR, there are still questions to be answered. What is the target cell of $2 \mathrm{D}$ nanomaterials? Whether the $2 \mathrm{D}$ nanomaterials directly interact with endothelial cells to drive the proliferation and capillary-forming responses? Or macrophages internalize the 2D nanomaterials to initiate the HIF-1a/VEGF signaling axis, and thereby attract endothelial cell migration? Whether the $2 \mathrm{D}$ nanomaterials need to be released from the scaffold to facilitate angiogenesis, or do they take effect in situ? Whether the protein within the regeneration microenvironment envelop the $2 \mathrm{D}$ nanomaterials to form the "protein corona" and whether the protein-adsorbed nanomaterials change their original biological activities (Wan et al., 2015)?

Moreover, the concentration of 2D nanomaterials should be taken into consideration when fabricating artificial nerve scaffolds. Many nanostructures can facilitate angiogenesis when administered at low dose, but at high dose, they will inhibit new vessel formation (Kargozar et al., 2020). This bimodal effect could be explained by that the low dose nanomaterial triggers moderate level of reactive oxygen species and thereby activates the downstream pro-angiogenesis signaling. However, high dose nanomaterial causes cytotoxicity and compromises cell activity, thereby contributes to the antiangiogenesis effects.

\section{PERSPECTIVE}

Although researchers developed various neural regeneration devices to treat PNI, long-range nerve defect remains a huge challenge (Qian et al., 2018a; Zhao et al., 2018; Chen et al., 2020; Qian et al., 2020b; Yan et al., 2021). The possibility for axons to travel through the chamber of a non-functionalized nerve scaffold is under $50 \%$ when the nerve defect exceeds $1 \mathrm{~cm}$ in rodents (Wieringa et al., 2018). Insufficient vascularization is observed in the long-range nerve defect model and it contributes to poor regeneration outcomes (Farber et al., 2016). With the development of 2D nanomaterials, the compromised vasculature formation could be remedied. Thus, 2D nanomaterials serve as a potential solution to long-range nerve defects.

Apart from angiogenesis, emphasis should also be placed upon the metabolic disturbance after peripheral nerve injury. Recent studies revealed the metabolically regulatory effects of certain biomaterials and the bioenergetic-active materials substantially accelerated tissue regeneration (Liu et al., 2020; Ma et al., 2019). Thus, the incorporation of bioenergetic active substrate into $2 \mathrm{D}$ nanomaterial nerve scaffold is poised to be an efficient and effective way to enhance the performance of conventional nerve scaffolds.

PNIs result in over 8.5 million restricted activity days and almost five million bed or disability days per year. In the US alone, over 200,000 peripheral nerve repair procedures are performed annually (Kehoe et al., 2012). So there is a great need for nerve regeneration devices. Since the mid-1980s, the FDA have approved several devices based on natural and synthetic materials to repair nerve defects, however, the therapeutic efficiency remains unsatisfactory. A preclinical assessment was carried out to examine the long-term biosafety and proregeneration effects of the graphene loaded nerve conduits (Qian et al., 2021a). The successful translation of these functionalized nerve conduits can meet the huge clinical demand and indirectly relieve the social and economic burden caused by PNIs.

\section{AUTHOR CONTRIBUTIONS}

YQ conceptualized the study. CC, CF, and ZY reviewed the literature and designed the figure and table. $\mathrm{ZY}$ drafted the manuscript. YQ, GR, and CF revised the manuscript. All authors read and approved the final version.

\section{FUNDING}

The study was sponsored by the Projects of National Natural Science Foundation of China (grant nos. 82002290, and 81830076), the Shanghai Sailing Program (no. 20YF1436000), Shanghai Engineering Research Center for Orthopaedic Material Innovation and Tissue Regeneration (no. 20DZ2254100), Municipal Hospital Newly-developing Cutting-edge Technologies Joint Research Program of Shanghai Shenkang 
Hospital Development Center (no. SHDC12018130), Special Fund for Research on People's Livelihood (Medical Treatment and Public Health) of Shanghai Pudong Science, Technology and Economic Commission Scientific and Technological Development Fund (no. PKJ2018-Y52), and Shanghai Pudong Health Commission Special Program for Clinical Research in the Health Industry (no. PW2018E-01).

\section{REFERENCES}

Babetto, E., Wong, K. M., and Beirowski, B. (2020). A Glycolytic Shift in Schwann Cells Supports Injured Axons. Nat. Neurosci. 23 (10), 1215-1228. doi:10.1038/ s41593-020-0689-4

Cattin, A.-L., and Lloyd, A. C. (2016). The Multicellular Complexity of Peripheral Nerve Regeneration. Curr. Opin. Neurobiol. 39, 38-46. doi:10.1016/ j.conb.2016.04.005

Cattin, A.-L., Burden, J. J., Van Emmenis, L., Mackenzie, F. E., Hoving, J. J. A., Garcia Calavia, N., et al. (2015). Macrophage-Induced Blood Vessels Guide Schwann Cell-Mediated Regeneration of Peripheral Nerves. Cell 162 (5), 1127-1139. doi:10.1016/j.cell.2015.07.021

Chen, X., Ge, X., Qian, Y., Tang, H., Song, J., Qu, X, et al. (2020). Electrospinning Multilayered Scaffolds Loaded with Melatonin and $\mathrm{Fe}_{3} \mathrm{O}_{4}$ Magnetic Nanoparticles for Peripheral Nerve Regeneration. Adv. Funct. Mater. 30, 2004537. doi:10.1002/adfm.202004537

Chen, X., Liu, C., Huang, Z., Pu, X., Shang, L., Yin, G., et al. (2019). Preparation of Carboxylic Graphene Oxide-composited Polypyrrole Conduits and Their Effect on Sciatic Nerve Repair under Electrical Stimulation. J. Biomed. Mater. Res. 107 (12), 2784-2795. doi:10.1002/jbm.a.36781

Chen, J., Lu, L., Zhang, C., Zhu, X., and Zhuang, S. (2021). Endothelial Dysfunction and Transcriptome Aberration in Mouse Aortas Induced by Black Phosphorus Quantum Dots and Nanosheets. Nanoscale 13 (19), 9018-9030. doi:10.1039/ d1nr01965a

Cheng, Y., Xu, Y., Qian, Y., Chen, X., Ouyang, Y., and Yuan, W.-E. (2020). 3D Structured Self-Powered PVDF/PCL Scaffolds for Peripheral Nerve Regeneration. Nano Energy 69, 104411. doi:10.1016/j.nanoen.2019.104411

Cho, Y., Shin, J. E., Ewan, E. E., Oh, Y. M., Pita-Thomas, W., and Cavalli, V. (2015). Activating Injury-Responsive Genes with Hypoxia Enhances Axon Regeneration through Neuronal HIF-1 $\alpha$. Neuron 88 (4), 720-734. doi:10.1016/j.neuron.2015.09.050

Cibecchini, G., Veronesi, M., Catelani, T., Bandiera, T., Guarnieri, D., and Pompa, P. P. (2020). Antiangiogenic Effect of Graphene Oxide in Primary Human Endothelial Cells. ACS Appl. Mater. Inter. 12 (20), 22507-22518. doi:10.1021/ acsami.0c03404

Clements, M. P., Byrne, E., Camarillo Guerrero, L. F., Cattin, A.-L., Zakka, L., Ashraf, A., et al. (2017). The Wound Microenvironment Reprograms Schwann Cells to Invasive Mesenchymal-like Cells to Drive Peripheral Nerve Regeneration. Neuron 96 (1), 98-114.e7. doi:10.1016/j.neuron.2017.09.008

Convertino, D., Fabbri, F., Mishra, N., Mainardi, M., Cappello, V., Testa, G., et al. (2020). Graphene Promotes Axon Elongation through Local Stall of Nerve Growth Factor Signaling Endosomes. Nano Lett. 20 (5), 3633-3641. doi:10.1021/acs.nanolett.0c00571

Fang, X., Guo, H., Zhang, W., Fang, H., Li, Q., Bai, S., et al. (2020). Reduced Graphene Oxide-GelMA-PCL Hybrid Nanofibers for Peripheral Nerve Regeneration. J. Mater. Chem. B 8 (46), 10593-10601. doi:10.1039/d0tb00779j

Farber, S. J., Hoben, G. M., Hunter, D. A., Yan, Y., Johnson, P. J., Mackinnon, S. E., et al. (2016). Vascularization is Delayed in Long Nerve Constructs Compared with Nerve Grafts. Muscle Nerve 54, 319-321. doi:10.1002/mus.25173

Fei, R., Li, W., Li, J., and Yang, L. (2015). Giant Piezoelectricity of Monolayer Group IV Monochalcogenides: SnSe, SnS, GeSe, and GeS. Appl. Phys. Lett. 107 (17), 173104. doi:10.1063/1.4934750

Fine, E. G., Valentini, R. F., Bellamkonda, R., and Aebischer, P. (1991). Improved Nerve Regeneration through Piezoelectric VinylidenefluorideTrifluoroethylene Copolymer Guidance Channels. Biomaterials 12 (8), 775-780. doi:10.1016/0142-9612(91)90029-a

\section{ACKNOWLEDGMENTS}

The authors appreciate the support from Base for Interdisciplinary Innovative Talent Training, Shanghai Jiao Tong University and Youth Science and Technology Innovation Studio of Shanghai Jiao Tong University School of Medicine.

Han, S. M., Baig, H. S., and Hammarlund, M. (2016). Mitochondria Localize to Injured Axons to Support Regeneration. Neuron 92 (6), 1308-1323. doi:10.1016/j.neuron.2016.11.025

Hobson, M. I., Brown, R., Green, C. J., and Terenghi, G. (1997). Inter-relationships between Angiogenesis and Nerve Regeneration: a Histochemical Study. Br. J. Plast. Surg. 50 (2), 125-131. doi:10.1016/s0007-1226(97)91325-4

Jessen, K. R., and Mirsky, R. (2016). The Repair Schwann Cell and its Function in Regenerating Nerves. J. Physiol. 594 (13), 3521-3531. doi:10.1113/JP270874

Jiang, H., Qian, Y., Fan, C., and Ouyang, Y. (2020). Polymeric Guide Conduits for Peripheral Nerve Tissue Engineering. Front. Bioeng. Biotechnol. 8, 582646. doi:10.3389/fbioe.2020.582646

Kargozar, S., Baino, F., Hamzehlou, S., Hamblin, M. R., and Mozafari, M. (2020). Nanotechnology for Angiogenesis: Opportunities and Challenges. Chem. Soc. Rev. 49 (14), 5008-5057. doi:10.1039/c8cs01021h

Kehoe, S., Zhang, X. F., and Boyd, D. (2012). FDA Approved Guidance Conduits and Wraps for Peripheral Nerve Injury: a Review of Materials and Efficacy. Injury 43 (5), 553-572. doi:10.1016/j.injury.2010.12.030

Koo, J., MacEwan, M. R., Kang, S.-K., Won, S. M., Stephen, M., Gamble, P., et al. (2018). Wireless Bioresorbable Electronic System Enables Sustained Nonpharmacological Neuroregenerative Therapy. Nat. Med. 24 (12), 1830-1836. doi:10.1038/s41591-018-0196-2

Lee, Y., Morrison, B. M., Li, Y., Lengacher, S., Farah, M. H., Hoffman, P. N., et al. (2012). Oligodendroglia Metabolically Support Axons and Contribute to Neurodegeneration. Nature 487 (7408), 443-448. doi:10.1038/ nature 11314

Lee, T.-H., Yen, C.-T., and Hsu, S.-h. (2020). Preparation of PolyurethaneGraphene Nanocomposite and Evaluation of Neurovascular Regeneration. ACS Biomater. Sci. Eng. 6 (1), 597-609. doi:10.1021/acsbiomaterials.9b01473

Li, Y., Shen, Q., Shen, J., Ding, X., Liu, T., He, J., et al. (2021). Multifunctional Fibroblasts Enhanced via Thermal and Freeze-Drying Post-treatments of Aligned Electrospun Nanofiber Membranes. Adv. Fiber Mater. 3 (1), 26-37. doi:10.1007/s42765-020-00059-3

Lin, H., Gao, S., Dai, C., Chen, Y., and Shi, J. (2017). A Two-Dimensional Biodegradable Niobium Carbide (MXene) for Photothermal Tumor Eradication in NIR-I and NIR-II Biowindows. J. Am. Chem. Soc. 139 (45), 16235-16247. doi:10.1021/jacs.7b07818

Lin, P., Pan, C., and Wang, Z. L. (2018). Two-dimensional Nanomaterials for Novel Piezotronics and Piezophototronics. Mater. Today Nano 4, 17-31. doi:10.1016/ j.mtnano.2018.11.006

Liu, H., Du, Y., St-Pierre, J. P., Bergholt, M. S., Autefage, H., and Wang, J. (2020). Bioenergetic-Active Materials Enhance Tissue Regeneration by Modulating Cellular Metabolic State. Sci. Adv. 6 (13), eaay7608. doi:10.1126/ sciadv.aay7608

Liu, Y., Wu, F., Ding, Y., Zhu, B., Su, Y., and Zhu, X. (2019). Preparation and Characterization of Paclitaxel/Chitosan Nanosuspensions for Drug Delivery System and Cytotoxicity Evaluation In Vitro. Adv. Fiber Mater. 1 (2), 152-162. doi:10.1007/s42765-019-00012-z

Luo, Y., Wang, X., and Cao, Y. (2021). Transcriptomic Analysis Suggested the Involvement of Impaired Lipid Droplet Biogenesis in Graphene Oxide-Induced Cytotoxicity in Human Umbilical Vein Endothelial Cells. Chemico-Biol. Interact. 333, 109325. doi:10.1016/j.cbi.2020.109325

Ma, C., Kuzma, M. L., Bai, X., and Yang, J. (2019). Biomaterial-Based Metabolic Regulation in Regenerative Engineering. Adv. Sci. 6 (19), 1900819. doi:10.1002/ advs.201900819

Min, Q., Parkinson, D. B., and Dun, X. P. (2021). Migrating Schwann Cells Direct Axon Regeneration within the Peripheral Nerve Bridge. Glia 69 (2), 235-254. doi:10.1002/glia.23892 
Norahan, M. H., Amroon, M., Ghahremanzadeh, R., Mahmoodi, M., and Baheiraei, N. (2019). Electroactive Graphene Oxide-Incorporated Collagen Assisting Vascularization for Cardiac Tissue Engineering. J. Biomed. Mater. Res. 107 (1), 204-219. doi:10.1002/jbm.a.36555

Pampaloni, N. P., Lottner, M., Giugliano, M., Matruglio, A., D’Amico, F., Prato, M., et al. (2018). Single-layer Graphene Modulates Neuronal Communication and Augments Membrane Ion Currents. Nat. Nanotech 13 (8), 755-764. doi:10.1038/s41565-018-0163-6

Patrón, L. A., and Zinsmaier, K. E. (2016). Mitochondria on the Road to Power Axonal Regeneration. Neuron 92 (6), 1152-1154. doi:10.1016/ j.neuron.2016.12.007

Qian, Y., Song, J., Zhao, X., Chen, W., Ouyang, Y., Yuan, W., et al. (2018a). 3D Fabrication with Integration Molding of a Graphene Oxide/Polycaprolactone Nanoscaffold for Neurite Regeneration and Angiogenesis. Adv. Sci. 5 (4), 1700499. doi:10.1002/advs.201700499

Qian, Y., Song, J., Zheng, W., Zhao, X., Ouyang, Y., Yuan, W., et al. (2018b). 3D Manufacture of Gold Nanocomposite Channels Facilitates Neural Differentiation and Regeneration. Adv. Funct. Mater. 28 (14), 1707077. doi:10.1002/adfm.201707077

Qian, Y., Zhao, X., Han, Q., Chen, W., Li, H., and Yuan, W. (2018c). An Integrated Multi-Layer 3D-Fabrication of PDA/RGD Coated Graphene Loaded PCL Nanoscaffold for Peripheral Nerve Restoration. Nat. Commun. 9 (1), 323. doi:10.1038/s41467-017-02598-7

Qian, Y., Cheng, Y., Cai, J., Zhao, X., Ouyang, Y., Yuan, W.-E., et al. (2019a). Advances in Electrical and Magnetic Stimulation on Nerve Regeneration. Regener. Med. 14 (10), 969-979. doi:10.2217/rme-2018-0079

Qian, Y., Cheng, Y., Ouyang, Y., Yuan, W.-E., and Fan, C. (2019b). Multilayered Spraying and Gradient Dotting of Nanodiamond-Polycaprolactone Guidance Channels for Restoration of Immune Homeostasis. NPG Asia Mater. 11 (1), 36. doi:10.1038/s41427-019-0136-8

Qian, Y., Han, Q., Zhao, X., Li, H., Yuan, W.-E., and Fan, C. (2019c). Asymmetrical 3D Nanoceria Channel for Severe Neurological Defect Regeneration. iScience 12, 216-231. doi:10.1016/j.isci.2019.01.013

Qian, Y., Yuan, W.-E., Cheng, Y., Yang, Y., Qu, X., and Fan, C. (2019d). Concentrically Integrative Bioassembly of a Three-Dimensional Black Phosphorus Nanoscaffold for Restoring Neurogenesis, Angiogenesis, and Immune Homeostasis. Nano Lett. 19 (12), 8990-9001. doi:10.1021/ acs.nanolett.9b03980

Qian, Y., Cheng, Y., Song, J., Xu, Y., Yuan, W. E., Fan, C., et al. (2020). MechanoInformed Biomimetic Polymer Scaffolds by Incorporating Self-Powered Zinc Oxide Nanogenerators Enhance Motor Recovery and Neural Function. Small 16 (32), 2000796. doi:10.1002/smll.202000796

Qian, Y., Wang, X., Song, J., Chen, W., Chen, S., Jin, Y., et al. (2021a). Preclinical Assessment on Neuronal Regeneration in the Injury-Related Microenvironment of Graphene-Based Scaffolds. NPJ Regen. Med. 6 (1), 31. doi:10.1038/s41536-021-00142-2

Qian, Y., Xu, Y., Yan, Z., Jin, Y., Chen, X., Yuan, W.-E., et al. (2021b). Boron Nitride Nanosheets Functionalized Channel Scaffold Favors Microenvironment Rebalance Cocktail Therapy for Piezocatalytic Neuronal Repair. Nano Energy 83, 105779. doi:10.1016/j.nanoen.2021.105779

Qian, Y., Lin, H., Yan, Z., Shi, J., and Fan, C. (2021c). Functional Nanomaterials in Peripheral Nerve Regeneration: Scaffold Design, Chemical Principles and Microenvironmental Remodeling. Mater. Today. doi:10.1016/ j.mattod.2021.09.014

Qian, Y., Yao, Z., Wang, X., Cheng, Y., Fang, Z., Yuan, W. E, et al. (2020b). (-)-Epigallocatechin Gallate-Loaded Polycaprolactone Scaffolds Fabricated Using a 3D Integrated Moulding Method Alleviate Immune Stress and Induce Neurogenesis. Cell Prolif. 53, e12730. doi:10.1111/cpr.12730

Ray, W. Z., and Mackinnon, S. E. (2010). Management of Nerve Gaps: Autografts, Allografts, Nerve Transfers, and End-To-Side Neurorrhaphy. Exp. Neurol. 223 (1), 77-85. doi:10.1016/j.expneurol.2009.03.031

Reis, J., Frias, C., Canto e Castro, C., Botelho, M. L., Marques, A. T., Simões, J. A. O., et al. (2012). A New Piezoelectric Actuator Induces Bone Formation in Vivo: A Preliminary Study. J. Biomed. Biotechnol. 2012, 613403. doi:10.1155/ 2012/613403

Rinholm, J. E., and Bergersen, L. H. (2012). Neuroscience: The Wrap that Feeds Neurons. Nature 487 (7408), 435-436. doi:10.1038/487435a
Rohaizad, N., Mayorga-Martinez, C. C., Fojtů, M., Latiff, N. M., and Pumera, M. (2021). Two-dimensional Materials in Biomedical, Biosensing and Sensing Applications. Chem. Soc. Rev. 50 (1), 619-657. doi:10.1039/d0cs00150c

Scheib, J., and Höke, A. (2013). Advances in Peripheral Nerve Regeneration. Nat. Rev. Neurol. 9 (12), 668-676. doi:10.1038/nrneurol.2013.227

Sidney, L. E., Branch, M. J., Dunphy, S. E., Dua, H. S., and Hopkinson, A. (2014). Concise Review: Evidence for $\mathrm{CD} 34$ as a Common Marker for Diverse Progenitors. Stem Cells 32 (6), 1380-1389. doi:10.1002/stem.1661

Sondell, M., Sundler, F., and Kanje, M. (2000). Vascular Endothelial Growth Factor Is a Neurotrophic Factor Which Stimulates Axonal Outgrowth through the Flk-1 Receptor. Eur. J. Neurosci. 12 (12), 4243-4254. doi:10.1046/j.0953-816x.2000.01326.x

Tao, J., Shen, W., Wu, S., Liu, L., Feng, Z., Wang, C., et al. (2015). Mechanical and Electrical Anisotropy of Few-Layer Black Phosphorus. ACS Nano 9 (11), 11362-11370. doi:10.1021/acsnano.5b05151

Taylor, C. A., Braza, D., Rice, J. B., and Dillingham, T. (2008). The Incidence of Peripheral Nerve Injury in Extremity Trauma. Am. J. Phys. Med. Rehabil. 87 (5), 381-385. doi:10.1097/PHM.0b013e31815e6370

Trimarco, A., and Taveggia, C. (2020). Schwann cell energy to die for. Nat. Neurosci. 23 (10), 1179-1181. doi:10.1038/s41593-020-00707-2

Veys, K., Fan, Z., Ghobrial, M., Bouché, A., García-Caballero, M., Vriens, K., et al. (2020). Role of the GLUT1 Glucose Transporter in Postnatal CNS Angiogenesis and Blood-Brain Barrier Integrity. Circ. Res. 127 (4), 466-482. doi:10.1161/ circresaha.119.316463

Wan, S., Kelly, P. M., Mahon, E., Stöckmann, H., Rudd, P. M., Caruso, F., et al. (2015). The "sweet" Side of the Protein corona: Effects of Glycosylation on Nanoparticle-Cell Interactions. ACS Nano 9 (2), 2157-2166. doi:10.1021/ nn506060q

Wang, J., Cheng, Y., Chen, L., Zhu, T., Ye, K., Jia, C., et al. (2019). In Vitro and In Vivo Studies of Electroactive Reduced Graphene Oxide-Modified Nanofiber Scaffolds for Peripheral Nerve Regeneration. Acta Biomater. 84, 98-113. doi:10.1016/j.actbio.2018.11.032

Webber, C. A., Christie, K. J., Cheng, C., Martinez, J. A., Singh, B., Singh, V., et al. (2011). Schwann Cells Direct Peripheral Nerve Regeneration through the Netrin-1 Receptors, DCC and Unc5H2. Glia 59 (10), 1503-1517. doi:10.1002/ glia.21194

Wieringa, P. A., Gonçalves de Pinho, A. R., Micera, S., van Wezel, R. J. A., and Moroni, L. (2018). Biomimetic Architectures for Peripheral Nerve Repair: A Review of Biofabrication Strategies. Adv. Healthc. Mater. 7, 1701164. doi:10.1002/ adhm.201701164

Wierzbicki, M., Hotowy, A., Kutwin, M., Jaworski, S., Bałaban, J., Sosnowska, M., et al. (2020). Graphene Oxide Scaffold Stimulates Differentiation and Proangiogenic Activities of Myogenic Progenitor Cells. Ijms 21 (11), 4173. doi:10.3390/ijms21114173

Xue, D., Chen, E., Zhong, H., Zhang, W., Wang, S., Joomun, M. U., et al. (2018). Immunomodulatory Properties of Graphene Oxide for Osteogenesis and Angiogenesis. Int. J. Nanomed. 13, 5799-5810. doi:10.2147/ijn.S170305

Yan, Z., and Qian, Y. (2020). Rectify the Injury-Induced Microenvironment Imbalance in Peripheral Nerve Repair. Aml 11 (10), 20101562. doi:10.5185/ amlett.2020.101562

Yan, Z., Qian, Y., and Fan, C. (2021). Biomimicry in 3D Printing Design: Implications for Peripheral Nerve Regeneration. Regen. Med. 16 (7), 683-701. doi:10.2217/rme-2020-0182

Yan, J., Wu, R., Liao, S., Jiang, M., and Qian, Y. (2020). Applications of Polydopamine-Modified Scaffolds in the Peripheral Nerve Tissue Engineering. Front. Bioeng. Biotechnol. 8, 590998. doi:10.3389/ fbioe.2020.590998

Yao, X., Qian, Y., and Fan, C. (2021). Electroactive Nanomaterials in the Peripheral Nerve Regeneration. J. Mater. Chem. B 9 (35), 6958-6972. doi:10.1039/ d1tb00686j

Zhang, D., Yao, Y., Duan, Y., Yu, X., Shi, H., Nakkala, J. R., et al. (2020). SurfaceAnchored Graphene Oxide Nanosheets on Cell-Scale Micropatterned Poly(d,lLactide-Co-Caprolactone) Conduits Promote Peripheral Nerve Regeneration. ACS Appl. Mater. Inter. 12 (7), 7915-7930. doi:10.1021/acsami.9b20321

Zhang, J., Tan, B., Zhang, X., Gao, F., Hu, Y., Wang, L., et al. (2021). Atomically Thin Hexagonal Boron Nitride and its Heterostructures. Adv. Mater. 33 (6), 2000769. doi:10.1002/adma.202000769 
Zhao, X., Qian, Y., Cheng, Y., Guo, X., and Yuan, W. E. (2018). One-Pot Construction of a Twice-Condensed pDNA Polyplex System for Peripheral Nerve Crush Injury Therapy. Biomater. Sci. 6 (8), 2059-2072. doi:10.1039/ c8bm00356d

Conflict of Interest: The authors declare that the research was conducted in the absence of any commercial or financial relationships that could be construed as a potential conflict of interest.

Publisher's Note: All claims expressed in this article are solely those of the authors and do not necessarily represent those of their affiliated organizations, or those of the publisher, the editors and the reviewers. Any product that may be evaluated in this article, or claim that may be made by its manufacturer, is not guaranteed or endorsed by the publisher.

Copyright (C) 2021 Yan, Chen, Rosso, Qian and Fan. This is an open-access article distributed under the terms of the Creative Commons Attribution License (CC BY). The use, distribution or reproduction in other forums is permitted, provided the original author(s) and the copyright owner(s) are credited and that the original publication in this journal is cited, in accordance with accepted academic practice. No use, distribution or reproduction is permitted which does not comply with these terms. 